\title{
Quantifying the burden of cardiovascular diseases among people living with HIV in sub-Saharan Africa: findings from a modeling study for Uganda
}

Alexander Kintu', David Sando', David Guwatudde ${ }^{2}$, Silver Bahendeka ${ }^{3}$, Peter C Kawungezi ${ }^{4}$, Gerald Mutungi ${ }^{5}$,Winnie Muyindike ${ }^{6}$, Nicolas Menzies ${ }^{1}$, Samson Okello ${ }^{7}$, Goodarz Danaei ${ }^{8}{ }^{\text {a }}$, Stéphane Verguet ${ }^{1}{ }^{b}$

1 Department of Global Health and Population, Harvard T.H. Chan School of Public Health, Boston, Massachusetts, USA, ${ }^{2}$ Department of Epidemiology and Biostatistics, School of Public Health, Makerere University College of Health Sciences, Kampala, Uganda, ${ }^{3}$ Department of Internal Medicine, St. Francis Nsambya Hospital, Kampala, Uganda, ${ }^{4}$ Department of Community Health, Mbarara University of Science and Technology, Mbarara, Uganda, ${ }^{5}$ Department of Non-Communicable Diseases Prevention and Control, Ministry of Health, Kampala, Uganda, ${ }^{6}$ Department of Internal Medicine, Mbarara University of Science and Technology, Mbarara, Uganda, 7 Department of Global Health and Population, Harvard T.H. Chan School of Public Health, Boston, Massachusetts, USA; Department of Internal Medicine, Mbarara University of Science and Technology, Mbarara, Uganda, ${ }^{8}$ Department of Global Health and Population, Harvard T.H. Chan School of Public Health, Boston, Massachusetts, USA; Department of Epidemiology, Harvard T.H. Chan School of Public Health, Boston MA, USA

Keywords: sub-saharan africa, cardiovascular disease, antiretroviral therapy, ncds, hiv

https://doi.org/10.29392/001c.14377

\section{Journal of Global Health Reports}

Vol. 4, 2020

\section{Background}

The burden of non-communicable diseases (NCDs) is rapidly increasing in low- and middle-income countries, but remains largely unknown among people living with HIV (PLWH) in most sub-Saharan African countries.

\section{Methods}

We estimated the proportion of PLWH in Uganda with raised blood pressure and high total cholesterol, and used a modified cardiovascular disease (CVD) risk prediction model (Globorisk) to assess the 10-year risk of atherosclerotic cardiovascular disease using individual-level data on cardiometabolic risk factors, population-level data on HIV prevalence and ART coverage, and the impact of HIV on blood pressure and cholesterol.

\section{Results}

Among PLWH aged 30 to 69 years, the prevalence of raised blood pressure was 30\% (95\% uncertainty range, $U R=27-33 \%)$ in women and $26 \%$ in men ( $95 \% \mathrm{UR}=23-29 \%)$. The predicted mean 10-year CVD risk was 5\% for HIV-infected women, and 6\% for HIV-infected men. Five percent $(n=41,000)$ of PLWH may experience a CVD event from 2016 to 2025 with an estimated 38\% of these events being fatal. Full ART coverage would have little effect on the predicted number of CVD cases.

\section{Conclusions}

Despite having a high prevalence of raised blood pressure, the burden of atherosclerotic CVD among PLWH in Uganda remains low. ART programs should prioritize routine screening and treatment of raised blood pressure. An approach of using HIV treatment delivery platforms to deliver care for NCDs may miss the larger burden of disease among HIV-uninfected individuals that are not routinely seen at health facilities.

Increased access to antiretroviral therapy (ART) for people living with HIV (PLWH) has substantially improved survival in countries with a high HIV prevalence. ${ }^{1}$ In Uganda, ART coverage reached $70 \%$ by $2017^{2}$ while life expectancy at birth increased from 45 years in 2004 (the year ART services were initially rolled out) to 62 years in $2017 .{ }^{3}$ In the meantime, there has been an increase in the burden of noncommunicable diseases (NCDs) in most of sub-Saharan Africa. ${ }^{4}$ With better survival and subsequent ageing, PLWH are faced with the increasing risk of NCDs, ${ }^{5,6}$ predominantly

\footnotetext{
a Co-senior author

b Co-senior author
} 
cardiovascular diseases (CVD). ${ }^{7}$ This has partly been attributed to inflammatory processes in HIV infection that are associated with an increased risk for CVD. ${ }^{8}$ In addition, as a result of increased ART coverage, PLWH increasingly have similar cardiometabolic risk profiles as those of HIV-uninfected individuals. ${ }^{9}$

Several studies in high-income countries found a higher CVD risk in PLWH compared with HIV-uninfected individuals. ${ }^{7}$ A pooled analysis of these studies estimated a $79 \%$ increased risk for myocardial infarction due to HIV infection and more than double the risk for stroke. ${ }^{7}$ In sub-Saharan Africa, studies comparing CVD risk by HIV status remain limited. Most studies are cross-sectional comparisons of traditional CVD risk factors by HIV status and have concluded that PLWH have more favorable CVD risk profiles. ${ }^{9}$ A systematic review estimated that PLWH in sub-Saharan Africa have on average $2 \cdot 3 \mathrm{~kg} / \mathrm{m}^{2}$ lower body mass index (BMI) than HIV-uninfected individuals, and their systolic blood pressure is on average $5.6 \mathrm{mmHg}$ lower. ${ }^{9}$ Longitudinal studies assessing CVD events are also sparse in sub-Saharan Africa due to challenges establishing these cohorts and in documenting CVD events. A case-control study from Tanzania found that HIV infection was associated with five times higher odds of stroke. ${ }^{10}$

Furthermore, national estimates on the prevalence of CVD risk factors and CVD risk in PLWH are not available in most of sub-Saharan Africa where countries do not have nationally representative surveys that jointly assess HIV/ART status and cardiometabolic risk factors and lack complete vital registration systems. In the absence of primary data on hard CVD endpoints, risk prediction models are a practical alternative but most of these models were developed using data from high-income countries that are not applicable to PLWH in sub-Saharan Africa. In addition, most models do not account for the direct effect of HIV on CVD, ${ }^{8}$ an effect that is independent of changes in risk factors like blood pressure, diabetes and total cholesterol. Excluding this effect would potentially underestimate CVD risk among HIVinfected individuals as was shown in a Ugandan study that predicted lower CVD risk in PLWH than in matched HIV-uninfected controls. ${ }^{11}$ This comparison was based on predictions made with Framingham and American Heart Association's pooled cohort risk scores, neither of which include this direct effect. Other studies have used the Data collection on Adverse Effects of Anti-HIV Drugs Study (D:A:D) prediction model which takes into account the effects of low immunity (CD4 cell count) and specific ART drugs on CVD risk. ${ }^{11,12}$ However, the D:A:D model was also developed for high-income countries and has not been recalibrated for use in sub-Saharan Africa. ${ }^{13}$

To fill this gap, we developed a microsimulation model to quantify the proportion of PLWH in Uganda with raised blood pressure and high total cholesterol, and used a risk prediction model calibrated for Uganda that takes into account the direct effect of HIV on CVD, to predict the burden of atherosclerotic CVD (stroke and coronary heart disease) among PLWH.

\section{METHODS}

We used various data sources to create a nationally rep- resentative sample of Ugandans aged 30 to 69 years, with estimates on traditional CVD risk factors (systolic blood pressure, diastolic blood pressure, total cholesterol, diabetes and smoking), as well as HIV and ART status. We then quantified the proportion of individuals with raised blood pressure and high total cholesterol using standard clinical thresholds. Thereafter, we used values on systolic blood pressure, total cholesterol, diabetes, smoking status, as well as age, sex and HIV status to predict each individual's 10-year risk for fatal and non-fatal CVD.

\section{DATA SOURCES}

Individual-level values on systolic blood pressure, diastolic blood pressure, total cholesterol, diabetes and smoking were available from the nationally representative 2014 Uganda STEPS Survey. ${ }^{14}$ Age and sex-disaggregated estimates for HIV prevalence were taken from the 2016 Uganda Population Based HIV Impact Assessment Survey (supplementary Table S1). ${ }^{15}$ We derived ART coverage estimates by sex for 2016 from a report by Uganda's Ministry of Health. ${ }^{16}$

\section{EFFECT OF HIV AND ART ON CVD RISK FACTORS}

HIV infection and ART have both been associated with changes in traditional CVD risk factors. ${ }^{9}$ We identified from a pooled analysis of sub-Saharan African studies, the mean differences in systolic blood pressure, diastolic blood pressure and fasting blood glucose by HIV and ART status. ${ }^{9} \mathrm{We}$ updated findings of this pooled analysis by including recently published articles (Table 1).

\section{CREATING A NATIONALLY REPRESENTATIVE SAMPLE}

We created a nationally representative sample of adults aged 30 to 69 years with individual-level data on HIV/ART status and on traditional CVD risk factors. This enabled us to take into account the direct effect of HIV on CVD, as well as the effects of HIV and ART on CVD that are mediated through traditional CVD risk factors. We included individuals aged 30 to 39 years because CVD events have been observed to occur at younger ages in HIV-infected individuals ${ }^{17}$; we did not include individuals older than 69 years because this age group represents a small population in Uganda (1\% in 2016) ${ }^{15}$ and was not included in the STEPS survey. As HIV status is not assessed in the STEPS survey, we used data on HIV prevalence and ART coverage to impute each individual's HIV and ART status by five-year age groups and sex, to create three categories of individuals: HIV-uninfected, HIV-infected not on ART, and HIV-infected on ART. We thereafter used data on the distributions of traditional CVD risk factors in the STEPS survey by age group and sex, mean differences of these risk factors by HIV and ART status (Table 1), as well as estimates for HIV prevalence and ART coverage to impute each individual's systolic blood pressure, diastolic blood pressure and total cholesterol levels. We kept the individual-level observed values from the STEPS survey for diabetes and smoking as there is no evidence for an effect of HIV, or ART on these two risk factors in Uganda (Figure 1). 
Table 1. Estimated mean differences in cardiometabolic risk factors by HIV and ART status

\begin{tabular}{|c|c|c|}
\hline \multirow{2}{*}{ Risk factor } & \multicolumn{2}{|c|}{ Estimate $^{\dagger}$} \\
\hline & HIV-infected vs. HIV-uninfected ${ }^{\ddagger}$ & ART-treated vs. ART-naïve ${ }^{\S}$ \\
\hline Systolic blood pressure, $\mathrm{mmHg}$ & $-5 \cdot 57(-7 \cdot 53,-3 \cdot 61)$ & $0.73(-2 \cdot 89,4 \cdot 35)$ \\
\hline Diastolic blood pressure, $\mathrm{mmHg} \|$ & $-3 \cdot 21(-4 \cdot 91,-1 \cdot 50)$ & $0.66(-0.44,1.76)$ \\
\hline Total blood cholesterol, mmol/L & $-0.39(-0.77,-0.02)$ & $0.57(0.25,0.89)$ \\
\hline Fasting blood glucose, $\mathrm{mmol} / \mathrm{L}^{\|}$ & $0 \cdot 11(-0 \cdot 59,0 \cdot 80)$ & $-0 \cdot 09(-0 \cdot 37,0 \cdot 20)$ \\
\hline \multicolumn{3}{|c|}{ 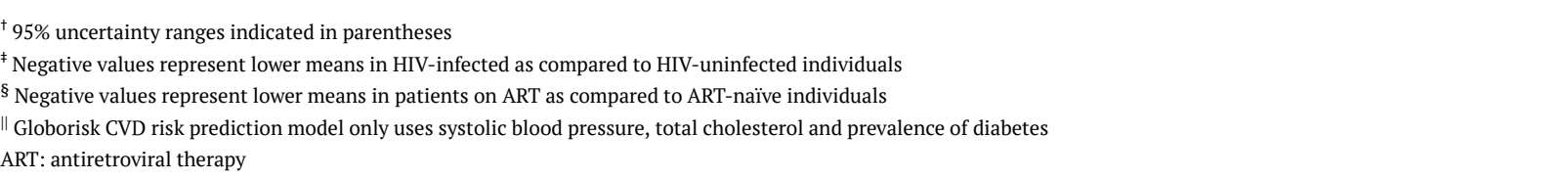 } \\
\hline
\end{tabular}

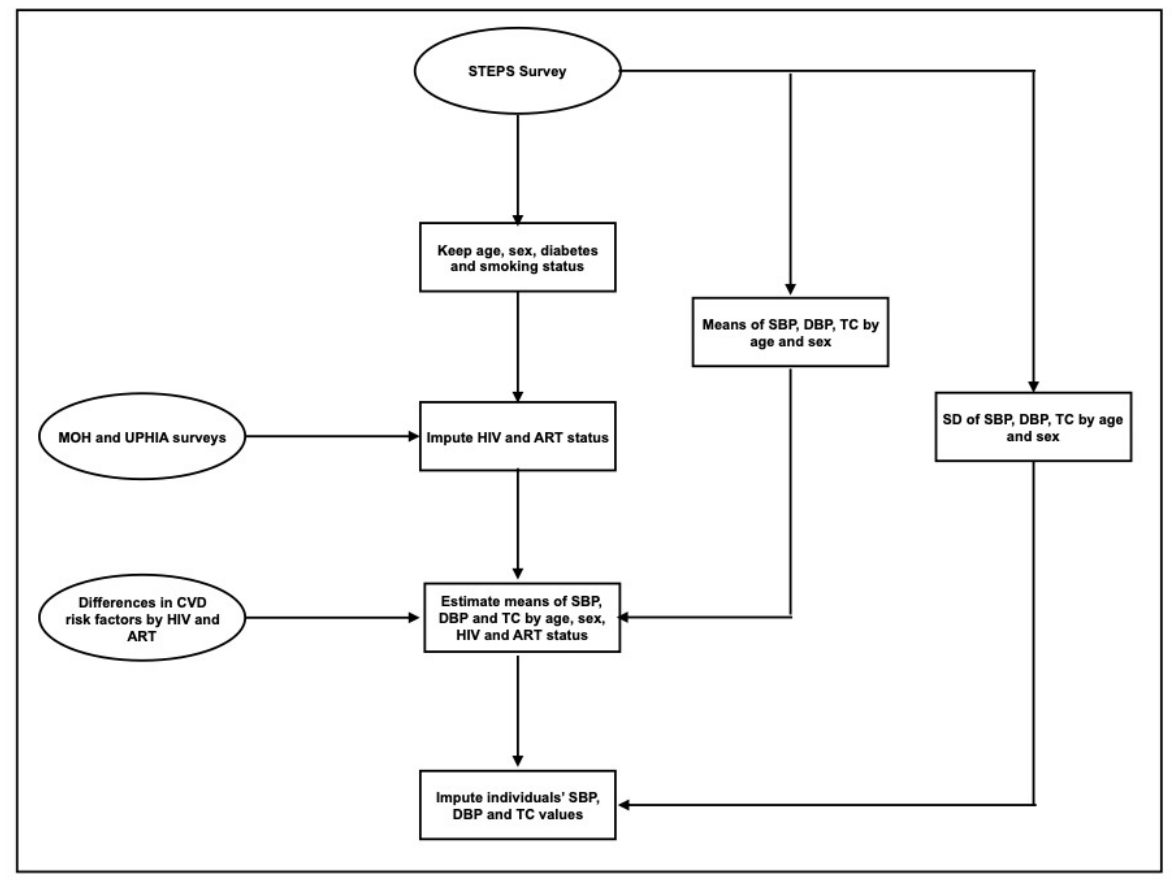

Figure 1: Flowchart for assigning CVD risk factors by HIV and ART status for individuals aged 30 to 69 years in Uganda.

STEPS: STEPWise approach to surveillance survey, MOH: Ministry of Health, UPHIA: Uganda Population Based HIV Impact Assessment Survey, SD: Standard deviation, CVD: cardiovascular diseases, ART: antiretroviral therapy, SBP: systolic blood pressure, DBP: diastolic blood pressure, TC: total cholesterol, DM: diabetes mellitus.

tObserved proportions of smokers and people with diabetes in the STEPS survey maintained because these two risk factors do not differ by HIV and ART status in Uganda.

\section{ESTIMATING CVD RISK AND CVD EVENTS IN HIV- INFECTED AND HIV-UNINFECTED INDIVIDUALS}

We modified the Globorisk model which was developed to estimate 10-year risk of stroke and coronary heart disease globally. ${ }^{18}$ This model uses pooled data from eight large prospective cohorts in the US and can be recalibrated for use in other countries. For Uganda, this was done by using age- and sex-specific CVD rates, and average CVD risk factor levels in the population (from the STEPS survey). We used CVD rates in Uganda from the World Health Organization (WHO) ${ }^{18}$ that are comparable to those of the Global Burden of Disease (GBD) study ${ }^{19}$ (Online Supplementary Document, Figure $S 1)$. We modified the risk prediction equation to include the direct effect of HIV on CVD that is not mediated through traditional CVD risk factors (Online Supplementary Document, Figure S2). To quantify the direct effect, we re-analyzed studies included in a recent meta-analysis. ${ }^{20}$ We identified studies in this meta-analysis with estimates of effect of HIV on CVD that is not mediated through metabolic risk factors, and pooled them separately for coronary heart disease and stroke. We combined the two pooled hazard ratios (HR) using the incidence rate of coronary heart disease and stroke in Uganda per the most recent estimates of the GBD study ${ }^{19}$ as weights to calculate a single HR for the direct effect of HIV on CVD (HR=1.63, 95\% confidence interval, $\mathrm{CI}=1 \cdot 31-1 \cdot 93)$. The modified model that includes the direct effect of HIV is: 


$$
\varphi_{i}(t)=\varphi_{0, s}(t) \exp \left[\sum_{l=1}^{4} \beta_{l}\left(X_{l, i}-\bar{X}_{l, s, t}\right)+\sum_{l=1}^{4} \delta_{l} t\left(X_{l, i}-\bar{X}_{l, s, t}^{\text {RESULTS }}\right.\right.
$$

$$
\left.\sum_{l=1}^{2} \gamma_{l} \operatorname{sex}_{i}\left(X_{l, i}-\bar{X}_{l, s, t}\right)+\alpha\left(H_{i}-\bar{H}_{s, t}\right)\right]
$$

A total of 6,975,373 adults aged 30-69 years were included

(1in the analysis and a majority (58\%) were women. The prevalence of HIV was $11 \%$ in women and $9 \%$ in men, and ART coverage was $77 \%$ for women and $54 \%$ for men.

The estimated mean systolic blood pressure in women was $129 \mathrm{mmHg}$ in those who were HIV-uninfected and 122 $\mathrm{mm} \mathrm{Hg}$ in HIV-infected individuals. For men, mean systolic blood pressure was $129 \mathrm{~mm} \mathrm{Hg}$ in those who were HIVuninfected and $123 \mathrm{mmHg}$ for HIV-infected counterparts. HIV infection was associated with a lower prevalence of raised blood pressure (Figure 2). The estimated mean total cholesterol levels were generally low, more so in men. The mean total cholesterol level in HIV-infected women was $4 \cdot 0$ $\mathrm{mmol} / \mathrm{L}$, and $3.9 \mathrm{mmol} / \mathrm{L}$ in women who were HIV-infected. For men, the mean total cholesterol level was $3.8 \mathrm{mmol} /$ $\mathrm{L}$ in those who were HIV-uninfected men, and $3.5 \mathrm{mmol} /$ $\mathrm{L}$ in HIV-infected individuals (Online Supplementary Document, Table S2).

The predicted mean 10-year risk for CVD was 5.0\% in HIV-infected women, 3.5\% in HIV-uninfected women, 6.3 $\%$ in HIV-infected men and $4.7 \%$ in HIV-uninfected men (Figure 3, panel A). Although HIV infection led to a higher estimated 10-year risk of CVD (Figure 3, panel $A$ ), a large proportion of HIV-infected individuals were at low risk for CVD as shown by a mean 10 -year CVD risk of $5 \%$ in women and $6 \%$ for men. $19 \%$ of HIV-infected women and $28 \%$ of HIV-infected men would meet criteria for primary prevention of CVD according to ACC/AHA guidelines (Figure 3, panel B). Our model predicted about 41,000 CVD cases to occur from 2016 to 2025 among HIV-infected individuals (19,000 in women and 22,000 in men), and about 320,000 cases among HIV-uninfected individuals (140,000 in women and 180,000 in men, Figure 4), a reflection of the respective numbers of HIV-infected and HIV-uninfected individuals in Uganda. Thirty-eight percent of the predicted CVD cases in HIV-infected individuals were fatal (44\% in women; 33\% in men). Among HIV-infected women, the predicted number of CVD cases was on average similar across the three age groups (ranging from 5,400 to 7,800 ), a pattern that is explained by the prevalence of HIV, the CVD rate and the population from each specific age group. For example, although HIV prevalence and population sizes were highest among women aged 30 to 44 years, higher CVD rates in women of older age groups resulted in almost similar CVD cases predicted for the three age groups. A different pattern was predicted for HIV-infected men: CVD cases doubled from 6,000 cases in men aged 30 to 44 years to over 12,000 cases in those aged 45 to 59 years because of the higher prevalence of HIV in older men $(5 \cdot 6 \%$ in men aged 30 to 34 years vs. $14.0 \%$ in those aged 45 to 49 years) and a higher CVD rate in older age groups. The predicted number of CVD cases subsequently declined to 3,000 cases in HIV-infected men aged 60 to 69 years due to the small population figures in this age group. Full ART coverage would have little impact on CVD risk due to already high ART coverage levels in 2016. For example, full ART coverage in 60 to 69 year-olds would result in a similar 10-year risk for CVD to the one that was predicted for ART coverage levels of 2016 (17\% vs. 16\% for women, and $18 \%$ vs. $20 \%$ for men). The predicted number 


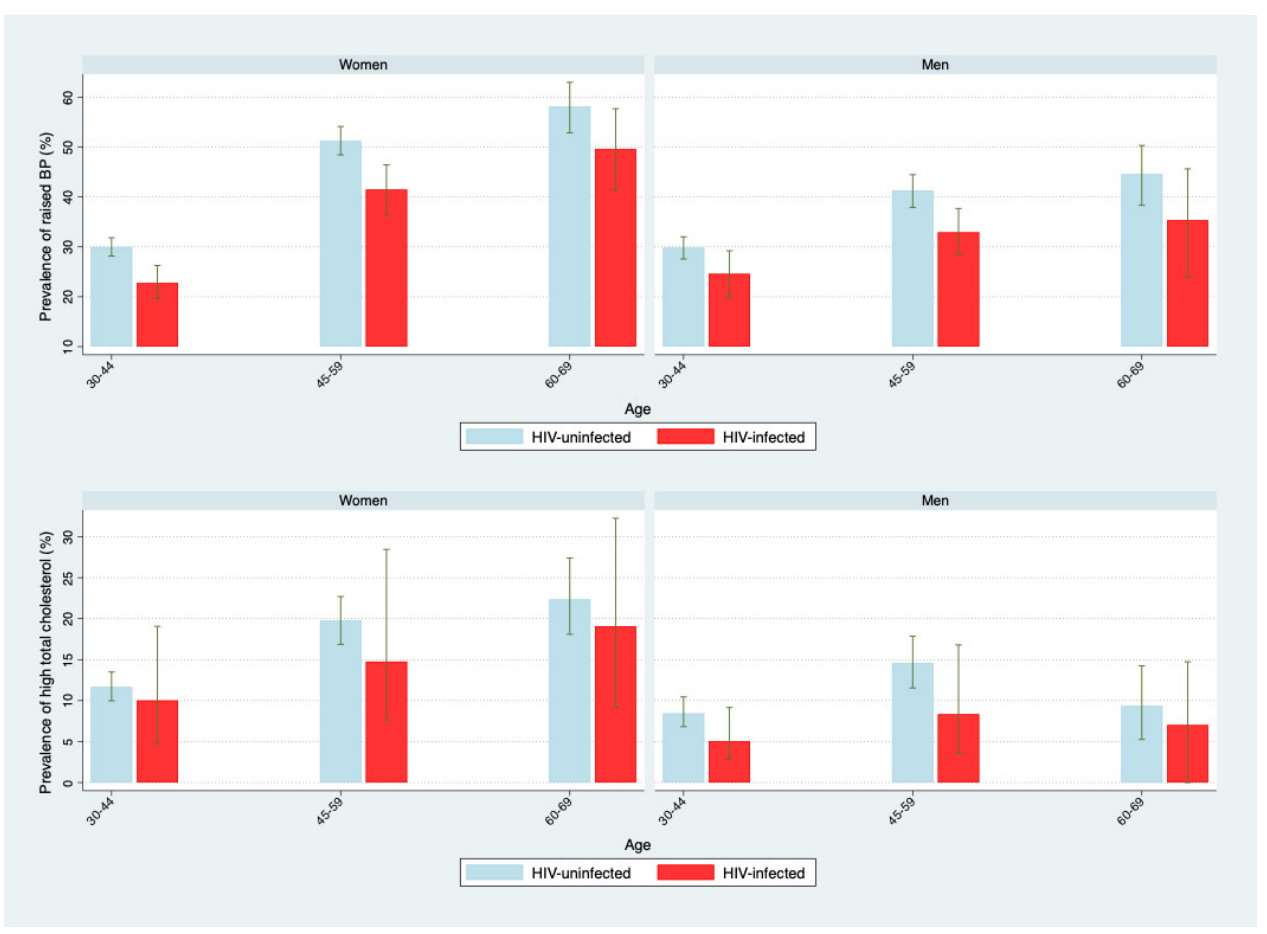

Figure 2: Estimated prevalence of raised blood pressure and high total cholesterol by HIV status in Uganda in 2016.

BP: blood pressure, TC: total cholesterol, ART: antiretroviral therapy.

Raised blood pressure defined as systolic blood pressure $\geqslant 140 \mathrm{mmHg}$, or diastolic blood pressure $\geqslant 90 \mathrm{mmHg}$, or being on antihypertensives.

High total cholesterol defined as total blood cholesterol $\geqslant 5.0 \mathrm{mmol} / \mathrm{L}$, or being on treatment for hyperlipidemia.

Note: $77 \%$ of HIV-infected women and $54 \%$ of HIV-infected men were on ART.

of CVD cases over ten years would also remain fairly unchanged (Online Supplementary Document, Figure S4).

\section{DISCUSSION}

We estimated the burden of CVD among PLWH in Uganda. Overall, we found that HIV infection was associated with a lower prevalence of raised blood pressure but a higher 10-year risk for CVD due to the harmful direct effect of HIV on CVD risk. Our findings further highlight that despite the higher risk of CVD in PLWH, the predicted burden of CVD is still low among this population with $5 \%(41,000)$ of all cases of stroke and CHD estimated to occur among PLWH from 2016 to 2025. To our knowledge, this is one of the first studies that uses a CVD risk prediction model calibrated for a specific sub-Saharan African country, and that includes the direct effect of HIV to quantify the national burden of CVD among PLWH.

Our estimates on traditional CVD risk factors (blood pressure and total cholesterol levels) are consistent with those of a study in western Uganda where PLWH had lower odds of hypertension than matched HIV-uninfected controls but where no difference was observed in mean total cholesterol levels by HIV status. ${ }^{23}$ Our estimates are representative of the CVD risk profile in Uganda in 2016: the lower proportion of raised blood pressure in HIV-infected individuals is in part due to immunosuppression in those who had not started ART by 2016 (23\% of women and $46 \%$ of men). ${ }^{16}$ These observed differences in CVD risk factors by HIV status are likely to reduce as more HIV-infected individuals start ART early. Yet, almost a third of adults living with HIV require treatment for raised blood pressure, a service that might not be readily available in most HIV treatment facilities. As per ACC/AHA guidelines, a sizable proportion (19\% of women and $28 \%$ of men) would also require statins for primary prevention of CVD, a therapy that is even more difficult to access in Uganda than treatment of raised blood pressure.

The estimated higher risk of CVD among HIV-infected individuals is due to the direct effect of HIV that outweighs the beneficial effect of having lower CVD risk factor levels when compared to HIV-uninfected individuals. We used a direct effect of HIV from a pooled estimate of studies in high-income countries. ${ }^{20}$ This HR is likely to be conservative because most HIV patients in these studies were on ART, a factor that was linked to lower risk for CVD in the Strategies for Management of Antiretroviral Therapy (SMART) trial. ${ }^{24}$ Our findings are consistent with previous studies that predicted low mean CVD risk among PLWH in Uganda. ${ }^{11,12}$ Only $17 \%$ of individuals (26\% males and $13 \%$ females) in one study had a 10 -year CVD risk that exceeded $5 \% .{ }^{12}$ However, even slightly higher risk of CVD associated with HIV infection is concerning, particularly in older ages, given the increasing longevity of HIV-infected individuals. In addition, annual new HIV infections in adults are still high in Uganda with over 83,000 new cases reported in $2015 .^{2}$ A continued trend of a high adult HIV incidence together with effects of aging on CVD risk factors will potentially lead to a larger burden of CVD among PLWH in the future.

The total number of CVD events predicted among HIVinfected and uninfected individuals over ten years $(362,000)$ 


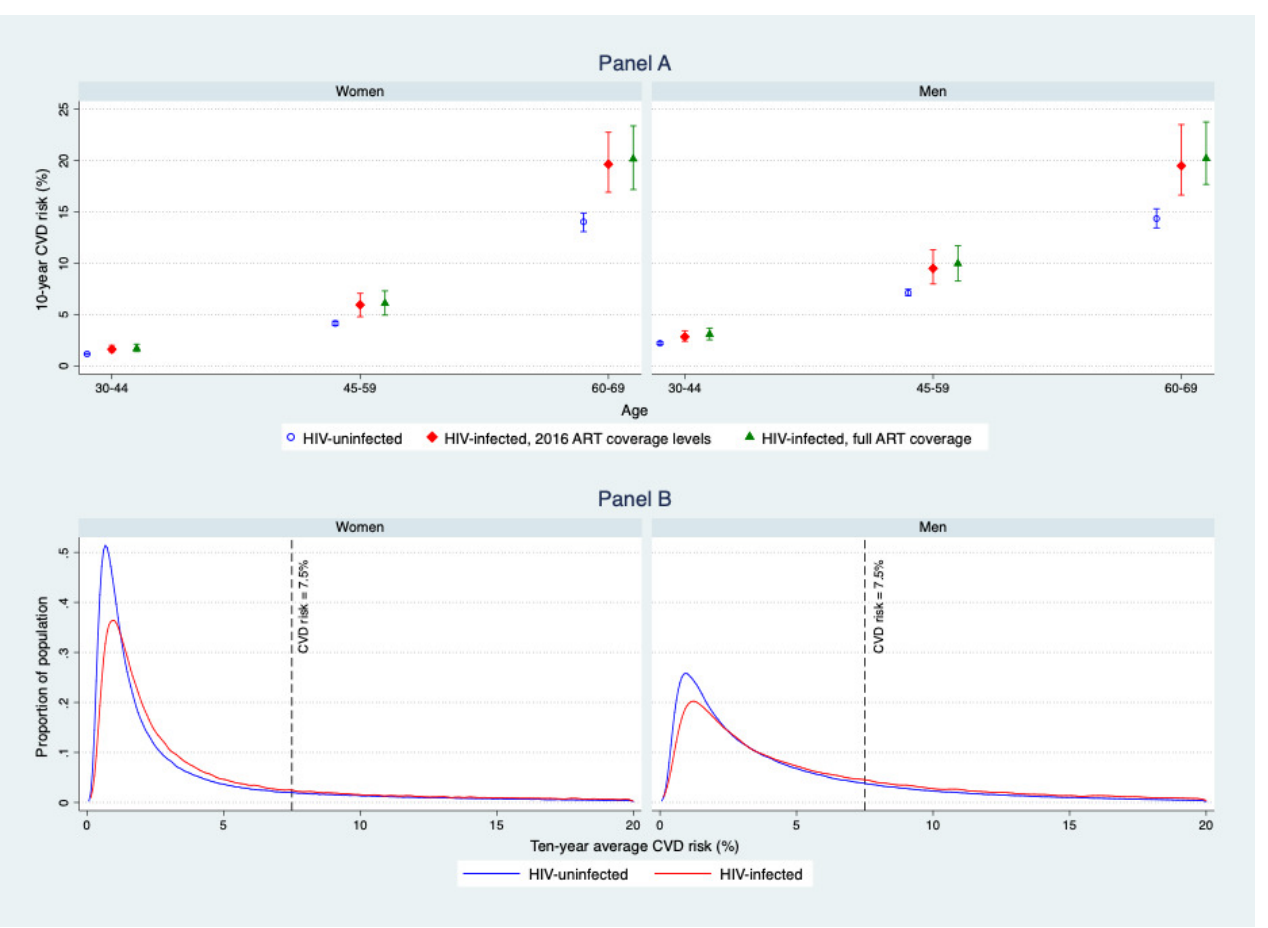

Figure 3: Estimated 10-year risk for fatal and non-fatal cardiovascular disease (CVD) in Uganda under different ART coverage scenarios (Panel A) and distributions of predicted 10-year risk for fatal and nonfatal CVD in the population by HIV status (Panel B)

ART: antiretroviral therapy.

Note: 77\% of HIV-infected women and 54\% of HIV-infected men were on ART in 2016.

Panel A: Estimated 10-year risk for cardiovascular disease by age, sex, HIV and ART status.

Panel B: Estimated distribution of CVD risk by sex and HIV status. Dotted vertical lines represent a 7.5\% 10-year risk threshold that would be used to identify high-risk individuals who would benefit from primary CVD prevention approaches.

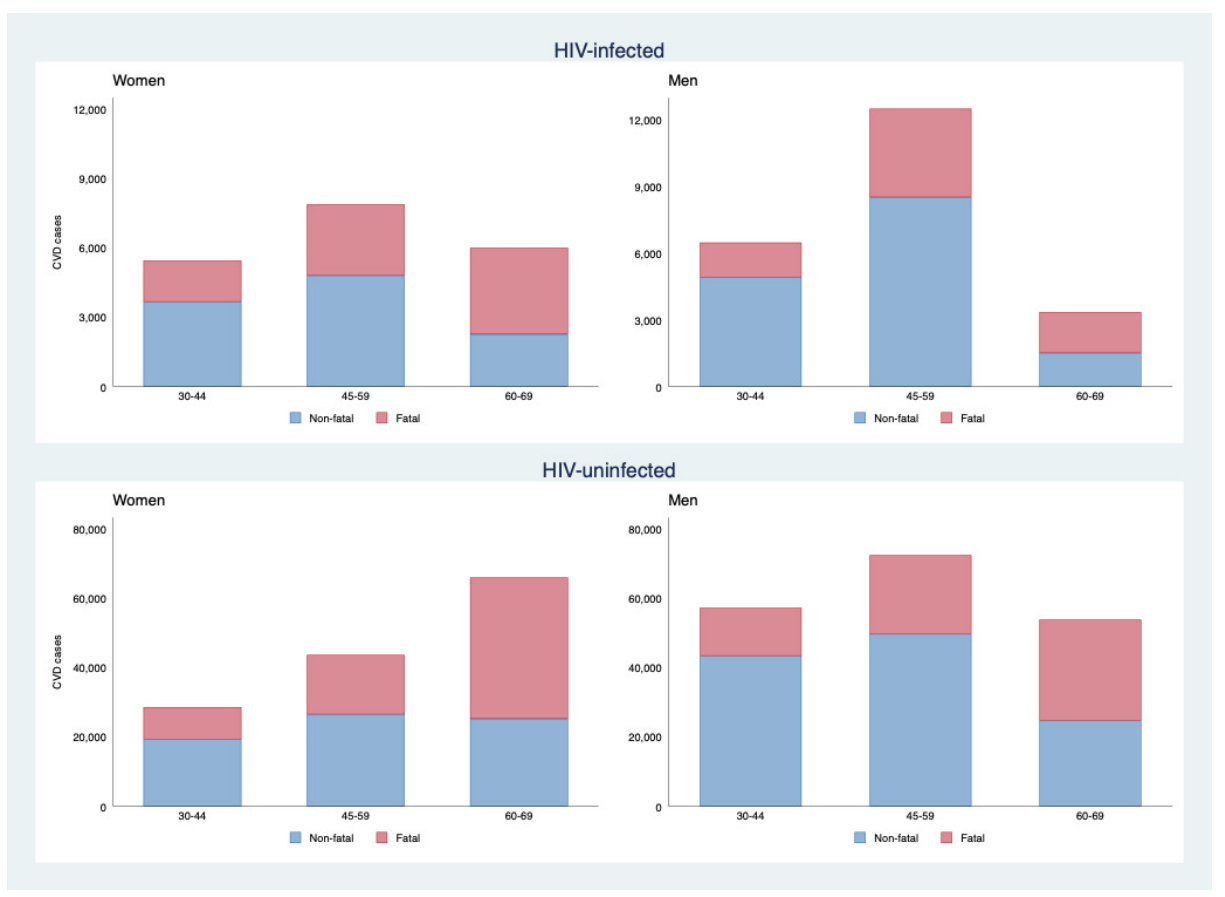

Figure 4: Estimated fatal and non-fatal cardiovascular disease (CVD) cases in Uganda from 2016 to 2025.

ART: antiretroviral therapy.

Note: $77 \%$ of HIV-infected women and 54\% of HIV-infected men were on ART

is comparable to estimates from the Global Burden of Dis- ease study. ${ }^{19}$ The overall burden of CVD is much higher 
among HIV-uninfected individuals due to their larger numbers in the population (adult HIV prevalence was 6\% in Uganda in 2016; Online Supplementary Document, Figure S3). The number of CVD events in PLWH (about 4,100 cases per year) is smaller than that of common opportunistic conditions in HIV. For example, an estimated 34,000 new cases of tuberculosis (TB) occur among PLWH in Uganda each year. ${ }^{25}$ The predicted number of CVD cases is also likely to increase as PLWH survive to older ages. Yet, our findings underscore the growing evidence that cardiovascular conditions are a leading non-HIV related cause of death in PLWH in sub-Saharan Africa. ${ }^{5}$ The high burden of CVD among HIV-uninfected individuals (about 32,000 cases per year) is alarming because the Uganda's current healthcare delivery system is unlikely to adequately address these large numbers. There has been increased advocacy for building onto existing HIV treatment platforms to deliver care for NCDs to the general population in countries with a high prevalence of HIV. ${ }^{26}$ There is however limited empirical evidence on how to achieve this, and this NCD-HIV integration approach is also likely to miss the larger burden of NCDs among HIV-uninfected individuals that are not routinely seen at health facilities.

We estimated that increasing ART coverage to 100\% would have little impact on the number of CVD cases among PLWH over ten years. This is due to the minimal impact of ART on blood pressure and total cholesterol (Table 1) and the moderately high ART coverage in PLWH in 2016 (77\% for women, $54 \%$ for men in Uganda) as a result of national policies that have promoted early initiation of ART. ${ }^{16}$ By 2011, in line with WHO recommendations, the Ministry of Health of Uganda enforced new guidelines requiring ART initiation at higher CD4 cell counts ( $\geqslant 350$ cells $/ \mathrm{mm}^{3}$ ) than before $^{27}$; and the test-and-treat initiative that was rolled out in 2016 will lead to even earlier ART initiation. ${ }^{27}$

Our analysis has several major strengths. First, we used nationally representative data on CVD risk factors, HIV prevalence and ART coverage to quantify the proportion of individuals with specific risk factors and each individual's 10 -year risk for CVD. We also took into account the effect of HIV and ART on CVD risk factors in sub-Saharan Africa by updating findings of a recent meta-analysis. ${ }^{9}$ Additionally, our analysis incorporated uncertainty in several parameters by accounting for their distributions and variations in the population. We used the Globorisk CVD prediction model as it could be easily calibrated to fit Uganda's CVD risk profile, ${ }^{18}$ to prevent over- or under-estimation of CVD risk when models developed for high-income countries are used in sub-Saharan African populations. ${ }^{28}$ We also revised the model to include the direct effect of HIV.

Nevertheless, our results should be interpreted with caution. First, as is conventional with most risk prediction scores, our risk equations did not consider competing risks. Although CVD has been shown to be a leading non-AIDS cause of death in PLWH in sub-Saharan Africa, ${ }^{5}$ there are other common causes of death like tuberculosis. Our risk prediction also did not include non-atherosclerotic CVD events like heart failure and sudden cardiac death that have been linked to HIV-infection. ${ }^{29,30}$ Second, our model did not incorporate the effects of low immunity and those of some antiretroviral drugs that have been linked to higher risk for CVD. ${ }^{8}$ Our analysis did not use age-disaggregated estimates of ART coverage and did not incorporate uncertainty in ART coverage because such data were not available. Lastly, as with other models, we are unable to validate the predicted CVD cases due to lack of hard clinical endpoints in this setting.

\section{CONCLUSIONS}

We found that the predicted burden of atherosclerotic CVD among PLWH remains low despite a high prevalence of raised blood pressure, and that the CVD burden is much higher among HIV-uninfected individuals. HIV treatment programs should prioritize screening and treatment of raised blood pressure so to avoid the missed opportunities that have been previously reported. ${ }^{31}$ Specific emphasis should be put on studying the clinical effects of Dolutegravir-based ART as countries in sub-Saharan Africa transition to this recommended first line therapy. Addressing the larger burden of CVD and other emerging NCDs among HIV-uninfected individuals will necessitate more empirical evidence on how to build onto existing HIV care and treatment platforms to deliver this care to the general population. 32

Acknowledgements: We gratefully acknowledge helpful comments from Kathryn Andrews and Katrina Ortblad on modeling risk factor levels by HIV status and those from Till Bärnighausen, Wafaie Fawzi, Shahin Lockman and Barnabas Nahwera on interpretation of the study's findings.

Funding: This study was supported by the US National Institutes of Health (NIH) Fogarty International Center through a grant from CRDF Global (Grant \# OISE-17-62965-1). The content is solely the responsibility of the authors and does not necessarily represent the official views of the US National Institutes of Health or CRDF Global.

Authorship contributions: SV conceived the study. DG, GM, $\mathrm{SB}, \mathrm{SO}, \mathrm{WM}$, and PK collected and provided data and advice for the analysis. AK built the simulation model, analyzed data, prepared results and wrote the first draft of the manuscript, under the supervision of SV and GD. NAM reviewed and provided advice on the simulation methods. All authors contributed to writing and reviewing the manuscript. AK and SV had final responsibility for submitting to publication.

Competing interests: The authors completed the Unified Competing Interest form at www.icmje.org/coi_disclosure.pdf (available upon request from the corresponding author), and declare no conflicts of interest.

\section{Correspondence to:}

Stéphane Verguet

Department of Global Health and Population

Harvard T.H. Chan School of Public Health 
665 Huntington Avenue

Boston MA 02115 USA.

verguet@hsph.harvard.edu

This is an open-access article distributed under the terms of the Creative Commons Attribution 4.0 International License (CCBY-4.0). View this license's legal deed at http://creativecommons.org/licenses/by/4.0 and legal code at http://creativecommons.org/licenses/by/4.0/legalcode for more information. 


\section{REFERENCES}

1. Nakagawa F, May M, Phillips A. Life expectancy living with HIV: Recent estimates and future implications. Curr Opin Infect Dis. 2013;26(1):17-25. doi:10.1097/aco.0b013e32835ba6b1

\section{Uganda AIDS Commission. THE UGANDA HIV AND} AIDS COUNTRY PROGRESS REPORT JULY 2015 -

JUNE 2016. Kampala, Uganda; 2016. https://www.una ids.org/sites/default/files/country/documents/UGA_20 17 countryreport.pdf.

3. World Bank. Life expectancy at birth, total (years) Uganda. https://data.worldbank.org/indicator/SP.DY N.LE00.IN?locations=UG. Accessed February 10, 2020.

4. Dalal S, Beunza JJ, Volmink J, et al. Noncommunicable diseases in sub-Saharan Africa: What we know now. Int J Epidemiol. 2011;40(4):885-901. do i:10.1093/ije/dyr050

5. Farahani M, Mulinder H, Farahani A, Marlink R. Prevalence and distribution of non-AIDS causes of death among HIV-infected individuals receiving antiretroviral therapy: A systematic review and metaanalysis. Int J STD AIDS. 2017;28(7):636-650. doi:10.1 $177 / 0956462416632428$

6. Haacker M, Bärnighausen T, Atun R. HIV and the growing health burden from noncommunicable diseases in Botswana: Modelling study. J Glob Health. 2019;9(1). doi:10.7189/jogh.09.010428

7. Shah ASV, Stelzle D, Lee KK, et al. Global Burden of Atherosclerotic Cardiovascular Disease in People Living with the Human Immunodeficiency Virus: A Systematic Review and Meta-Analysis. Circulation. 2018;138(11):1100-1112. doi:10.1161/circulationah a.117.033369

8. Gibellini D, Borderi M, Clò A, et al. HIV-related mechanisms in atherosclerosis and cardiovascular diseases. J Cardiovasc Med. 2013;14(11):780-790. do i:10.2459/jcm.0b013e3283619331

9. Dillon DG, Gurdasani D, Riha J, Ekoru K, Asiki G, Mayanja BN, et al. Association of HIV and ART with cardiometabolic traits in sub-Saharan Africa: A systematic review and meta-analysis. Int J Epidemiol. 2013;42(6):1754-1771.

10. Walker R, Whiting D, Unwin N, et al. Stroke incidence in rural and urban Tanzania: A prospective, community-based study. Lancet Neurol. 2010;9(8):786-792. doi:10.1016/s1474-4422(10)7014 4-7
11. Muiru AN, Bibangambah PM, Hemphill L, et al. Distribution and Performance of Cardiovascular Risk Scores in a Mixed Population of HIV-Infected and Community-Based HIV-Uninfected Individuals in Uganda. J Acquir Immune Defic Syndr. 2018;78(4):458-464. doi:10.1097/qai.00000000000016 96

12. Muyanja D, Muzoora C, Muyingo A, Muyindike W, Siedner MJ. High Prevalence of Metabolic Syndrome and Cardiovascular Disease Risk Among People with HIV on Stable ART in Southwestern Uganda. AIDS Patient Care and STDs. 2016;30(1):4-10. doi:10.1089/ apc.2015.0213

13. Boateng D, Agyemang C, Beune E, et al. Cardiovascular disease risk prediction in sub-Saharan African populations - Comparative analysis of risk algorithms in the RODAM study. Int J Cardiol. 2018;254:310-315. doi:10.1016/i.ijcard.2017.11.082

14. World Health Organization. A framework for surveillance: The WHO STEPwise approach to Surveillance of noncommunicable diseases (STEPS). 2003. https://www.who.int/ncd_surveillance/en/step s framework dec03.pdf.

15. Ministry of Health - Uganda. Uganda Population Based HIV Impact Survey. https://phia.icap.columbi a.edu/wp-content/uploads/2018/07/3430\%E2\%80\%A2 PHIA-Uganda-SS NEW.v14.pdf. Accessed November 12, 2019.

16. Ministry of Health - Uganda. Uganda HIV/AIDS Country Progress Report July 2016 - June 2017. Kampala, Uganda: Ministry of Health https://www.un aids.org/sites/default/files/country/documents/UGA 2 018 countryreport.pdf. Accessed August 17, 2020.

17. Boccara F, Lang S, Meuleman C, et al. HIV and coronary heart disease: Time for a better understanding. J Am Coll Cardiol. 2013;61(5):511-523. doi:10.1016/j.jacc.2012.06.063

18. Hajifathalian K, Ueda P, Lu Y, et al. A novel risk score to predict cardiovascular disease risk in national populations (Globorisk): A pooled analysis of prospective cohorts and health examination surveys. Lancet Diabetes Endocrinol. 2015;3(5):339-355. doi:1 $\underline{0.1016 / \mathrm{s} 2213-8587(15) 00081-9}$

19. GBD 2016 Disease and Injury Incidence and Prevalence Collaborators. Global, regional, and national incidence, prevalence, and years lived with disability for 328 diseases and injuries for 195 countries, 1990-2016: A systematic analysis for the Global Burden of Disease Study 2016. Lancet. 2017;390(10100):1211-1259. 
20. Gutierrez J, Albuquerque ALA, Falzon L. HIV infection as vascular risk: A systematic review of the literature and meta-analysis. Lin Y-J, ed. PLOS ONE. 2017;12(5):e0176686. doi:10.1371/journal.pone.0176 $\underline{686}$

21. Stone NJ, Robinson JG, Lichtenstein AH, et al. 2013 ACC/AHA Guideline on the Treatment of Blood Cholesterol to Reduce Atherosclerotic Cardiovascular Risk in Adults: a report of the American College of Cardiology/American Heart Association Task Force on Practice Guidelines. Circulation. 2013;129(25 suppl 2):S1-S45. doi:10.1161/01.cir.0000437738.63853.7a

22. World Population Prospects - Population Division. United Nations https://esa.un.org/unpd/wpp/Graphs/ DemographicProfiles/. Accessed April 11, 2018.

23. Okello S, Ueda P, Kanyesigye M, et al. Association between HIV and blood pressure in adults and role of body weight as a mediator: Cross-sectional study in Uganda. J Clin Hypertens. 2017;19(11):1181-1191. do $\mathrm{i}: 10.1111 / \mathrm{jch} .13092$

24. Strategies for Management of Antiretroviral Therapy (SMART) Study Group. CD4+ Count-Guided Interruption of Antiretroviral Treatment. $\mathrm{N}$ Engl J Med. 2006;355(22):2283-2296. doi:10.1056/nejmoa06 $\underline{2360}$

25. World Health Organization. GLOBAL TUBERCULOSIS REPORT 2018. Geneva; 2018. http://a pps.who.int/iris/bitstream/handle/10665/274453/978 9241565646-eng.pdf?ua=1. Accessed November 27, 2018.

26. El-Sadr WM, Goosby E. Building on the HIV platform: Tackling the challenge of noncommunicable diseases among persons living with HIV. AIDS. 2018;32(Suppl 1):S1-S3. doi:10.1097/ gad.0000000000001886
27. Ministry of Health - Uganda. Consolidated Guidelines for Prevention and Treatment of HIV in Uganda. Kampala, Uganda; 2016. http://library.healt h.go.ug/publications/hivaids/consolidated-guideline s-prevention-and-treatment-hiv-uganda. Accessed August 17, 2020.

28. Mosepele M, Hemphill LC, Palai T, et al. Cardiovascular disease risk prediction by the American College of Cardiology (ACC)/American Heart Association (AHA) Atherosclerotic Cardiovascular Disease (ASCVD) risk score among HIV-infected patients in sub-Saharan Africa. De Socio GV, ed. PLoS ONE. 2017;12(2):e0172897. doi:10.1371/ journal.pone.0172897

29. Freiberg MS, Chang C-CH, Skanderson M, et al. Association Between HIV Infection and the Risk of Heart Failure With Reduced Ejection Fraction and Preserved Ejection Fraction in the Antiretroviral Therapy Era. JAMA Cardiol. 2017;2(5):536-546. doi:1 0.1001/jamacardio.2017.0264

30. Tseng ZH, Secemsky EA, Dowdy D, et al. Sudden Cardiac Death in Patients with Human Immunodeficiency Virus Infection. J Am Coll Cardiol. 2012;59(21):1891-1896. doi:10.1016/j.jacc.2012.02.02 $\underline{4}$

31. Kalyesubula R, Kayongo A, Semitala FC, et al. Trends and level of control of hypertension among adults attending an ambulatory HIV clinic in Kampala, Uganda: A retrospective study. BMJ Glob Health. 2016;1(3):e000055. doi:10.1136/bmigh-201 6-000055

32. Vorkoper S, Kupfer LE, Anand N, et al. Building on the HIV chronic care platform to address noncommunicable diseases in sub-Saharan Africa: A research agenda. AIDS. 2018;32(Suppl 1):S107-S113. doi:10.1097/qad.0000000000001898 


\section{SUPPLEMENTARY MATERIALS}

\section{Online Supplementary Document}

Download: https://www.joghr.org/article/14377-quantifying-the-burden-of-cardiovascular-diseases-among-peopleliving-with-hiv-in-sub-saharan-africa-findings-from-a-modeling-study-for-uganda/attachment/39652.pdf 